\title{
Therapeutic strategies for type 2 diabetes mellitus patients with very high HbA1c: is insulin the only option?
}

\author{
Muhammad Abdul-Ghani, Ralph DeFronzo \\ Division of Diabetes, University of Texas Health Science Center at San Antonio, San Antonio, TX, USA \\ Correspondence to: Muhammad Abdul-Ghani, MD, PhD. Division of Diabetes, University of Texas Health Science Center at San Antonio, 7703 Floyd \\ Curl Drive, San Antonio, TX, USA. Email: abdulghani@uthscsa.edu. \\ Provenance: This is an invited Editorial commissioned by the Section Editor Kaiping Zhang (AME College, AME Group, China). \\ Comment on: Henry RR, Rosenstock J, Denham DS, et al. Clinical Impact of ITCA 650, a Novel Drug-Device GLP-1 Receptor Agonist, in \\ Uncontrolled Type 2 Diabetes and Very High Baseline HbA1c: The FREEDOM-1 HBL (High Baseline) Study. Diabetes Care 2018;41:613-9.
}

Submitted Nov 07, 2018. Accepted for publication Nov 08, 2018.

doi: $10.21037 / \mathrm{atm} .2018 .11 .15$

View this article at: http://dx.doi.org/10.21037/atm.2018.11.15

Approximately $40 \%$ of patients with type 2 diabetes mellitus (T2DM) will experience microvascular complications (1), and T2DM is the leading cause of renal failure and blindness in western countries (2-4). Two land mark studies, the Diabetes Control and Complications Trial (DCCT) (5) and United Kingdom Prospective Diabetes Study (UKPDS) (6), as well as many others (7-9) have documented that hyperglycemia is the major risk factor for microvascular complication and that the risk of developing those complications is directly related to the magnitude of hyperglycemia as measured by the HbA1c. Further, improving glucose control markedly reduces diabetic microvascular risk. Each $1 \%$ reduction in the HbA1c is associated with $\sim 40 \%$ reduction in the risk of development of retinopathy and nephropathy $(5,6)$. Therefore, maintaining good glycemic control (i.e., HbA1c < $7.0 \%$ ) while preventing microvascular complications is the mainstay of management of T2DM as well as T1DM and is recommended by all professional organizations (10).

Despite the irrefutable evidence of the importance of adequate glycemic control in reducing microvascular risk, approximately one half of T2DM patients fail to achieve the recommended American Diabetes Association (ADA) goal of glycemic control, i.e., HbA1c < $7.0 \%(11,12)$. Further, several studies have reported that $10-15 \%$ of T2DM patients in the US and elsewhere manifest HbAlc $>9.0 \%$ $(13,14)$, rendering them at very high risk for microvascular complications.

Because T2DM is lifelong disease, adherence to antiglycemic therapy is essential for the achievement of long-term glycemic control. Multiple studies have demonstrated that more than half of T2DM patients discontinue their antihyperglycemic medications within 12 months of initiating therapy $(15,16)$, and this discontinuation rate is even higher amongst patients receiving injectable agents, e.g., glucagon-like peptide-1 receptors agonists (GLP-1 RA) (17). Thus, improving adherence to antihyperglycemic therapy is an important unmet need in the management of T2DM. The study by Henry and colleagues (18) examined the impact of a novel drug delivery system, ITCA 650 , on glucose control in T2DM patients with very high HbA1c (mean $=10.8 \pm 0.7$, range, $10-12 \%)$. ITCA 650 is an osmotic pump of matchstick size which is placed underneath the skin and continuously delivers exenatide at a predetermined constant rate for 6-12 months. Placement of the device takes 10-15 minutes and involves a simple procedure that can be performed in the clinic under local anesthesia. Thus, once the device is placed, it ensures $100 \%$ adherence to therapy for the 6-12 months treatment period.

Henry and colleagues (18) enrolled sixty poorly controlled T2DM patients with HbA1c 10-12\% who did not qualify for the placebo-controlled trial because the baseline HbA1c was $>10 \%$ and a placebo-controlled study in this group was felt to be unethical. Other than diabetes, patients were in good general health and treated with lifestyle alone or lifestyle plus metformin with or without sulfonylurea. The ITCA 650 device that delivers $20 \mu \mathrm{g} /$ day 
was implanted for 13 weeks, at which time the device was replaced with another which delivered $60 \mu \mathrm{g} /$ day for additional 26 weeks.

The primary outcome of the study was the decrease in HbA1c from baseline to week 39. Rescue therapy was allowed in patients in whom the HbA1c did not decrease below $8.5 \%$ at week 26 . Fifty-two $(86.7 \%)$ patients completed the study. Six patients withdraw consent because of adverse events and two patients were lost to follow-up.

The mean decrease in $\mathrm{HbA1c}$ at 39 weeks was $2.8 \%$. Approximately half of the patients experienced $>3 \%$ reduction in $\mathrm{HbAlc}$, and one quarter experienced $>4 \%$ decrease in HbA1c. Moreover, despite very high initial HbA1c (HbA1c $>10 \%)$, approximately one quarter of patients achieved the ADA goal for glycemic control (i.e., HbA1c $<7.0 \%)$ at 39 weeks. The reduction in HbA1c produced by ITCA 650 is impressive when considering that $20 \%$ of participant were able to discontinue their baseline antihyperglycemic therapy and remain on exenatide monotherapy. Two factors contribute to the robust decrease in HbA1c. First, it is very well established that the higher the starting HbAlc, the greater is the decrease in HbAlc with all antidiabetic agents. Second, the GLP-1 RA are intrinsically potent glucose lowering agents and correct 6 components of the ominous octet $(19,20)$. Further, as pointed out by the authors, a major advantage of the ITCA 650 is that $100 \%$ adherence to therapy is ensured by ITCA 650 and this is rarely/never has been achieved in other clinical studies with injectable (daily or weekly) GLP-1 RA. Not pointed out by the investigators, however, is the potent rapid onset and durable effect of GLP-1 RA to reduce the HbA1c. This only can be achieved by improving beta cell function which is essential in providing durable reduction in HbA1c. Thus, normalization of beta cell function has been shown within 8 hours after a single injection of liraglutide (21) and persisted for up to 3 years with exenatide (22).

Despite the impressive decline in HbA1c $(2.8 \%$ decrease), the resultant HbA1c on mean would be $\sim 8.0 \%$ which still is above the goal recommended by the ADA/ European Association for the Study of Diabetes (EASD). However, the good news is that addition of a single oral antidiabetic agent, pioglitazone (23) or sodium/glucose cotransporter 2 (SGLT2) inhibitor, is likely to further reduce the HbA1c to the ADA goal of $<7.0 \%$.

Most clinical guidelines (10) recommend that T2DM patients with a HbA1c $>10 \%$ be started on insulin therapy, especially if symptoms (polyuria, polydipsia, nocturia and weight loss) symptoms of hyperglycemia are evident. However, this recommendation more is based on "expert opinion" than on objective evidence. The results of Henry et al. (18) demonstrate that ITCA 650 provides an alternative therapeutic option for patients with a HbA1c between $10-12 \%$. Further, the results of Henry et al. are consistent with a previous study from our group (23) which demonstrated that combination therapy with exenatide plus pioglitazone produced an even greater decrement in HbA1c $(-4.8 \%)$ in T2DM patients with a baseline HbA1c $=10-16 \%$ (mean HbA1c $=11.4 \%$ ). Further, the decrement in HbA1c produced by exenatide plus pioglitazone was significantly greater than that produced by basal/bolus insulin $(4.8 \% v s$. $3.8 \%, \mathrm{P}<0.001)$, and more patient achieved the ADA goal of glycemic control with exenatide plus pioglitazone $(70 \%$ vs. $35 \%$, respectively, $\mathrm{P}=0.003)$. Collectively, these results $(18,23)$ suggest that a GLP-1 RA alone or in combination with pioglitazone provide a very effective therapeutic option alternative to insulin for glucose control in patients with a HbA1c $>10 \%$. A major advantage of combined exenatide/ pioglitazone therapy is that these agents "normalize" glucose metabolism by correcting 7 of the 8 Ominous Octet including the two major core defects, insulin resistance and beta cell failure present in T2DM patients (19,24-26).

Although ITCA 650 was very effective in improving glycemic control, its effect on body weight was modest, $1.2 \mathrm{~kg}$ and it did not reach statistical significance. This result is surprising and is in contrast with other studies which have examined the effect of GLP-1 RA monotherapy, including exenatide, on glucose control in T2DM. These studies consistently have reported significant weight loss in the range of 3-5 kg over 52-104 weeks $(27,28)$. Because participants in the study had very poor glycemic control (HbA1c $=10-12 \%)$, it is likely that many patients had a mean day long plasma glucose concentration that was well above the threshold for renal glucose excretion (29). The robust reduction in HbA1c would have been expected to markedly reduce glucosuria, thereby, reducing urinary caloric loss and offsetting the intrinsic action of the GLP-1 RA to promote weight loss. Measurement of urinary glucose excretion before, during, and at the end of exenatide therapy could have added insight about the impact of ITCA 650 on energy balance and body weight in T2DM patients with very high initial HbA1c.

Lastly, the safety profile of ITCA 650 was consistent with that of other GLP-1 RAs $(27,28)$. Other than nausea, no other drug related adverse events were reported. And, similar to other GLP-1 RAs, the nausea was transient, and 
was observed in only $~ 20 \%$ of patients at the initiation of treatment and at 13 weeks when exenatide dose was escalated to $60 \mu \mathrm{g} /$ day.

\section{Acknowledgements}

None.

\section{Footnote}

Conflicts of Interest: R DeFronzo is on the Advisory Board of Intarcia and AstraZeneca, and received grand support from AstraZeneca. The other author has no conflicts of interest to declare.

\section{References}

1. He Z, King GL. Microvascular complications of diabetes. Endocrinol Metab Clin North Am 2004;33:215-38.

2. Shen Y, Cai R, Sun J, et al. Diabetes mellitus as a risk factor for incident chronic kidney disease and end-stagerenal disease in women compared with men: a systematic review and meta-analysis. Endocrine 2017;55:66-76.

3. Fox CS, Matsushita K, Woodward M, et al. Associations of kidney disease measures with mortality and end-stage renal disease in individuals with and without diabetes: a metaanalysis. Lancet 2012;380:1662-73.

4. Solomon SD, Chew E, Duh EJ, et al. Diabetic Retinopathy: A Position Statement by the American Diabetes Association. Diabetes Care 2017;40:412-8.

5. Nathan DM, Genuth S, Lachin J, et al. The effect of intensive treatment of diabetes on the development and progression of long-term complications in insulin-dependent diabetes mellitus. N Engl J Med 1993;329:977-86.

6. Intensive blood-glucose control with sulphonylureas or insulin compared with conventional treatment and risk of complications in patients with type 2 diabetes (UKPDS 33). UK Prospective Diabetes Study (UKPDS) Group. Lancet 1998;352:837-53.

7. Nakagami T, Kawahara R, Hori S, et al. Glycemic control and prevention of retinopathy in Japanese NIDDM patients. A 10-year follow-up study. Diabetes Care 1997;20:621-2.

8. Klein R, Klein BE, Moss SE. Relation of glycemic control to diabetic microvascular complications in diabetes mellitus. Ann Intern Med 1996;124:90-6.

9. Ohkubo $\mathrm{Y}$, , Kishikawa H, Araki E, et al. Intensive insulin therapy prevents the progression of diabetic microvascular complications in Japanese patients with non-insulindependent diabetes mellitus: a randomized prospective 6-year study. Diabetes Res Clin Pract 1995;28:103-17.

10. Davies MJ, D'Alessio DA, Fradkin J, et al. Management of hyperglycaemia in type 2 diabetes, 2018. A consensus report by the American Diabetes Association (ADA) and the European Association for the Study of Diabetes (EASD). Diabetologia 2018;61:2461-98.

11. Hoerger TJ, Segel JE, Gregg EW, et al. Is glycemic control improving in U.S. adults? Diabetes Care 2008;31:81-6.

12. Ali MK, Bullard KM, Saaddine JB, et al. Achievement of goals in U.S. diabetes care, 1999-2010. N Engl J Med 2013;368:1613-24.

13. Aronson R, Orzech N, Ye C, et al. Specialist-led diabetes registries and predictors of poor glycemic control in type 2 diabetes: insights into the functionally refractory patient from the LMC Diabetes Registry database. J Diabetes 2016;8:76-85.

14. Alatorre C, Fernández Landó L, Yu M. Treatment patterns in patients with type 2 diabetes mellitus treated with glucagon-like peptide-1 receptor agonists: higher adherence and persistence with dulaglutide compared with once-weekly exenatide and liraglutide. Diabetes Obes Metab 2017;19:953-61.

15. Cheung BM, Ong KL, Cherny SS, et al. Diabetes prevalence and therapeutic target achievement in the United States, 1999 to 2006. Am J Med 2009;122:443-53.

16. Egede LE, Gebregziabher M, Echols C, et al. Longitudinal effects of medication nonadherence on glycemic control. Ann Pharmacother 2014;48:562-70.

17. Buysman EK, Liu F, Hammer M, et al. Impact of medication adherence and persistence on clinical and economic outcomes in patients with type 2 diabetes treated with liraglutide: a retrospective cohort study. Adv Ther 2015;32:341-55.

18. Henry RR, Rosenstock J, Denham DS, et al. Clinical Impact of ITCA 650, a Novel Drug-Device GLP-1 Receptor Agonist, in Uncontrolled Type 2 Diabetes and Very High Baseline HbA1c: The FREEDOM-1 HBL (High Baseline) Study. Diabetes Care 2018;41:613-9.

19. Defronzo RA. Banting Lecture. From the triumvirate to the ominous octet: a new paradigm for the treatment of type 2 diabetes mellitus. Diabetes 2009;58:773-95.

20. Abdul-Ghani M, DeFronzo RA. Is It Time to Change the Type 2 Diabetes Treatment Paradigm? Yes! GLP-1 RAs Should Replace Metformin in the Type 2 Diabetes 
Algorithm. Diabetes Care 2017;40:1121-7.

21. Chang AM, Jakobsen G, Sturis J, et al. The GLP-1 derivative NN2211 restores beta-cell sensitivity to glucose in type 2 diabetic patients after a single dose. Diabetes 2003;52:1786-91.

22. Bunck MC, Cornér A, Eliasson B, et al. Effects of exenatide on measures of $\beta$-cell function after 3 years in metformin-treated patients with type 2 diabetes. Diabetes Care 2011;34:2041-7.

23. Abdul-Ghani M, Migahid O, Megahed A, et al. Efficacy of Exenatide Plus Pioglitazone Vs Basal/Bolus Insulin in T2DM Patients With Very High HbA1c. J Clin Endocrinol Metab 2017;102:2162-70.

24. Abdul-Ghani MA, Puckett C, Triplitt C, et al. Initial combination therapy with metformin, pioglitazone and exenatide is more effective than sequential add-on therapy in subjects with new-onset diabetes. Results from the Efficacy and Durability of Initial Combination Therapy for Type 2 Diabetes (EDICT): a randomized trial. Diabetes Obes Metab 2015;17:268-75.

25. Armato JP, DeFronzo RA, Abdul-Ghani M, et al. Successful treatment of prediabetes in clinical practice using physiological assessment (STOP DIABETES).

Cite this article as: Abdul-Ghani M, DeFronzo R. Therapeutic strategies for type 2 diabetes mellitus patients with very high HbA1c: is insulin the only option? Ann Transl Med 2018;6(Suppl 2):S95. doi: 10.21037/atm.2018.11.15
Lancet Diabetes Endocrinol 2018;6:781-9.

26. Abdul-Ghani M, Migahid O, Megahed A, et al. Combination Therapy With Exenatide Plus Pioglitazone Versus Basal/Bolus Insulin in Patients With Poorly Controlled Type 2 Diabetes on Sulfonylurea Plus Metformin: The Qatar Study. Diabetes Care 2017;40:325-31.

27. Singh S, Wright EE Jr, Kwan AY, et al. Glucagonlike peptide-1 receptor agonists compared with basal insulins for the treatment of type 2 diabetes mellitus: a systematic review and meta-analysis. Diabetes Obes Metab 2017;19:228-38.

28. Witkowski M, Wilkinson L, Webb N, et al. A Systematic Literature Review and Network Meta-Analysis Comparing Once-Weekly Semaglutide with Other GLP-1 Receptor Agonists in Patients with Type 2 Diabetes Previously Receiving 1-2 Oral Anti-Diabetic Drugs. Diabetes Ther 2018;9:1149-67.

29. Al-Jobori H, Daniele G, Cersosimo E, et al. Empagliflozin and Kinetics of Renal Glucose Transport in Healthy Individuals and Individuals With Type 2 Diabetes. Diabetes 2017;66:1999-2006. 